\title{
RECESSION METHODS IN MONOTONE VARIATIONAL HEMIVARIATIONAL INEQUALITIES
}

\author{
Samir Adly - Daniel Goeleven — Michel Théra
}

Dedicated to Professor Ky Fan on his eightieth birthday

\section{Introduction, notations and definitions}

Throughout the paper we use standard notations except special symbols introduced when they are defined. All spaces considered are Banach spaces whose norms are always denoted by $\|\cdot\|$. For any space $V$ we consider its dual space $V^{\star}$ equipped with the strong topology. We denote by $\langle\cdot, \cdot\rangle$ the duality pairing between $V$ and $V^{\star}$. Let $f: V \rightarrow \mathbb{R} \cup\{\infty\}$ be an extended-real-valued function. Identifying extended-real-valued functions with their epigraphs

$$
\text { epi } f=\{(x, \alpha) \mid x \in V, \alpha \in \mathbb{R} \text { and } \alpha \geq f(x)\}
$$

is a standard tool in convex analysis and in one-sided optimization theory. Also, those functions with closed epigraphs are precisely the lower semicontinuous functions on $V$, and as usual,

$$
\operatorname{dom} f:=\{x \in V \mid f(x)<\infty\} .
$$

We say that $f$ is proper if $\operatorname{dom} f$ is nonempty. In this case $\lim \sup f(x)$ and $\lim \inf f(x)$ denote the upper and lower limits of such (scalar) functions in the classical sense. Depending on context, the symbols $x \stackrel{\mathrm{s}}{\rightarrow} y$ and $x \rightarrow y$ mean,

1991 Mathematics Subject Classification. 47H19, 49J52, 70B15, 73 T05.

Key words and phrases. Minimax inequality, variational hemivariational inequality, recession function, monotone operator, Clarke's subgradient, asymptotic direction, robot hand grasping problem. 
respectively, that $x$ tends to $y$ with respect to the strong topology and the weak topology on $V$.

The theory of variational inequalities is now well established and one of the most famous result which has greatly contributed to its development is the Fan minimax principle (see [9] for the original proof). It should be observed that this principle is an immediate consequence of a two-function minimax theorem proved independently by Ben-El-Mechaiekh, Deguire \& Granas [6], Yen [27] and Simons [24]:

TheOREM 1. Let $C$ be a nonempty compact convex subset of a topological vector space and let $f, g: C \times C \rightarrow \mathbb{R}$ be two functions satisfying:

(i) $g \leq f$ on $C \times C$;

(ii) $f$ is quasiconcave in its first variable and $g$ is lower semicontinuous in its second variable.

Then

$$
\min _{y \in C} \sup _{x \in C} g(x, y) \leq \sup _{x \in C} f(x, x) .
$$

As another immediate consequence of the preceding theorem we also have:

Corollary 2. Let $C$ be a nonempty bounded closed convex subset of a real reflexive Banach space $V$. Let $f, g: C \times C \rightarrow \mathbb{R}$ be two functions satisfying:

(i) $g(x, y) \leq f(x, y)$ for all $x, y \in C$;

(ii) for each $y \in C$, the function $x \mapsto f(x, y)$ is concave;

(iii) for each $x \in C$, the function $y \mapsto g(x, y)$ is lower semicontinuous;

(iv) $f(x, x) \leq 0$ for all $x \in C$.

Then there exists $u \in C$ such that $g(v, u) \leq 0$ for all $v \in C$.

Indeed, either this minimax principle or one of its equivalent forms has been used by many authors in order to obtain existence theorems applicable to several classes of variational inequalities.

Our aim in this paper is to use this two-function minimax theorem in a new direction of research, namely, in the field of hemivariational inequalities, theory introduced and developed by P. D. Panagiotopoulos [21] since the early 80 s. The aim of this theory is the treatment of nonconvex, nonsmooth energy problems in mechanics. Since variational inequalities are based on the notion of convexity and are formulated for monotone multivalued boundary conditions and/or constitutive laws, they fail to apply to the problems listed above. The theory of variational hemivariational inequalities has been employed by P. D. Panagiatopoulos and his collaborators as a very efficient tool to describe the behavior of several complex structures, such as for instance the delamination problem in laminated composites, where the interaction between the laminae due to the binding material is described by means of a nonmonotone, possibly multivalued 
law. Such laws express a variety of limit phenomena related to the discontinuous loss of resistance at the surface. For complete references on the origin of the theory of hemivariational inequalities, a basic reference is the book of P. D. Panagiotopoulos [20].

Let us introduce further definitions. A functional $j: V \rightarrow \mathbb{R}$ is said to be locally Lipschitz if for each $x \in V$, there exist a neighbourhood $\mathcal{N}(x)$ of $x$ and a constant $k_{\mathcal{N}}>0$ such that

$$
|j(u)-j(v)| \leq k_{\mathcal{N}}\|u-v\|, \quad \forall u, v \in \mathcal{N} .
$$

We recall that an operator $A: V \rightarrow V^{*}$ is said to be monotone if

$$
\langle A u-A v, u-v\rangle \geq 0 \quad \forall u, v \in V,
$$

while $A$ is declared hemicontinuous if the functional $t \mapsto\langle A(u+t v), w\rangle$ is continuous on $[0,1]$ for all $u, v, w \in V$.

Let $K$ be a nonempty, closed and convex subset of a reflexive Banach space $V$. In order to simplify some computations we will assume that $0 \in K$. Throughout the paper, we assume that the assumptions $(\mathcal{H})$ described below are satisfied:

$\left(\mathrm{H}_{1}\right) \quad A: V \rightarrow V^{*}$ is a monotone and hemicontinuous operator;

$\left(\mathrm{H}_{2}\right) j: V \rightarrow \mathbb{R}$ is a locally Lipschitz function;

$\left(\mathrm{H}_{3}\right) \Phi: V \rightarrow \mathbb{R} \cup\{\infty\}$ is a proper, convex and lower semicontinuous function satisfying $\Phi(0)=0$;

$\left(\mathrm{H}_{4}\right) f$ is given in $V^{*}$.

For a locally Lipschitzian functional $j: V \rightarrow \mathbb{R}$, we denote by $j^{\circ}(u ; v)$ the Clarke generalized directional derivative of $j$ at $u$ in the direction $v$, that is,

$$
j^{\circ}(u ; v):=\limsup _{\substack{\lambda \rightarrow 0^{+} \\ w \rightarrow u}} \frac{j(w+\lambda v)-j(w)}{\lambda} .
$$

Recall also at this point that

$$
\bar{\partial} j(x):=\left\{x^{*} \in V^{*} \mid j^{\circ}(x ; y) \geq\left\langle x^{*}, y\right\rangle \forall y \in V\right\}
$$

denotes the generalized Clarke subdifferential [8].

Let $K$ be a closed convex subset of $V$. A vector $y$ is called a direction of recession in $K$ at $x$ if for each positive $t$ the vector $x+t y$ lies in $K$. The directions of recession are independent of $x$ and form a closed convex cone called the recession cone of $K$ :

$$
K_{\infty}:=\bigcap_{t>0}\left[\frac{K-x_{0}}{t}\right],
$$

where $x_{0}$ is arbitrary chosen in $K$. Equivalently, this amounts to saying that $x$ belongs to $K_{\infty}$ if and only if there exist sequences $\left\{t_{n}\right\}_{n \in \mathbb{N}}$ and $\left\{x_{n}\right\}_{n \in \mathbb{N}} \subseteq K$ such that $\lim _{n \rightarrow \infty} t_{n}=\infty$ and $x=\lim _{n \rightarrow \infty} t_{n}^{-1} x_{n}$. The notion of recession cone 
has been used to sharpen many of the classical results of convexity theory (see e.g. R. T. Rockafellar [23]).

Let $\Phi: V \rightarrow \mathbb{R} \cup\{\infty\}$ be a proper, convex and lower semicontinuous function. Then the behavior at infinity of $\Phi$ can be described by the recession function $\Phi_{\infty}$ of $\Phi$ which is defined by the formula

$$
\Phi_{\infty}(x):=\lim _{t \rightarrow \infty} \frac{\Phi\left(x_{0}+t x\right)-\Phi\left(x_{0}\right)}{t},
$$

where $x_{0}$ is taken arbitrary such that $\Phi\left(x_{0}\right)<\infty$. It is easily observed that

$$
\operatorname{epi} \Phi_{\infty}=\{\operatorname{epi} \Phi\}_{\infty} .
$$

In order to cover the case of nonconvex functionals, C. Baiocchi, G. Buttazzo, F. Gastaldi and F. Tomarelli [5] have introduced a more general concept of recession function. Let $\Psi: V \rightarrow \mathbb{R}$ be any functional. The recession function associated with $\Psi$ is defined by

$$
\Psi^{\infty}(x):=\inf \left\{\liminf _{n \rightarrow \infty} \Psi\left(t_{n} x_{n}\right) / t_{n} \mid t_{n} \rightarrow \infty, x_{n} \stackrel{\mathrm{s}}{\rightarrow} x\right\} .
$$

By a variational hemivariational inequality H.V.I. $(A, f, j, \Phi, k)$ we mean the problem

H.V.I. $(A, f, j, \Phi, K)$ : Find $u \in K$ such that

$$
\langle A u-f, v-u\rangle+j^{\circ}(u ; v-u)+\Phi(v)-\Phi(u) \geq 0, \quad \forall v \in K .
$$

Using Corollary 2, we begin with proving an existence theorem for the class of problems H.V.I. $(A, f, j, \Phi, K)$ involving a bounded set of constraints.

Lemma 3. Let $C$ be a nonempty bounded closed convex subset of $V$ such that $0 \in C$. Assume that the assumptions $(\mathcal{H})$ are satisfied. Then there exists $u \in C$ such that

$$
\langle A u-f, v-u\rangle+j^{\circ}(u ; v-u)+\Phi(v)-\Phi(u) \geq 0, \quad \forall v \in C .
$$

Proof. Set

$$
\begin{aligned}
& f(x, y):=\langle A y-f, y-x\rangle-j^{\circ}(y ; x-y)+\Phi(y)-\Phi(x), \\
& g(x, y):=\langle A x-f, y-x\rangle-j^{\circ}(y ; x-y)+\Phi(y)-\Phi(x) .
\end{aligned}
$$

The monotonicity of $A$ yields

$$
g(x, y) \leq f(x, y)
$$

For each $y \in C$, the map $x \mapsto j^{\circ}(y ; x)$ is convex [8; Proposition 2.1.1] and thus $x \mapsto f(x, y)$ is concave. The map $(x, y) \mapsto j^{\circ}(x ; y)$ is upper semicontinuous as a function of $(x, y)$ [8; Proposition 2.1.1] and therefore the function $y \mapsto g(x, y)$ is lower semicontinuous. 
We have $f(x, x)=0$ for all $x \in C$. Hence by virtue of Corollary 2, we obtain the existence of $u \in C$ such that $g(v, u) \leq 0$ for all $v \in C$. Equivalently, there exists $u \in C$ such that

$$
\langle A v-f, v-u\rangle+j^{\circ}(u ; v-u)+\Phi(v)-\Phi(u) \geq 0, \quad \forall v \in C .
$$

Let $y \in C$ be arbitrary. We set $v:=t y+(1-t) u, 0<t<1$. By putting $v$ in (1.1), we obtain

$$
\begin{array}{r}
\langle A(u+t(y-u)), t(y-u)\rangle+j^{\circ}(u ; t(y-u))+\Phi(u+t(y-u))-\Phi(u) \\
\geq\langle f, t(y-u)\rangle .
\end{array}
$$

Dividing (1.2) by $t>0$ and using the convexity of $\Phi$, we derive

$$
\langle A(u+t(y-u)), y-u\rangle+j^{\circ}(u ; y-u)+\Phi(y)-\Phi(u) \geq\langle f, y-u\rangle .
$$

Taking the limit as $t \rightarrow 0$ and using the hemicontinuity of $A,(1.3)$ yields

$$
\langle A u, y-u\rangle+j^{\circ}(u ; y-u)+\Phi(y)-\Phi(u) \geq\langle f, y-u\rangle .
$$

This ends the proof of the lemma.

In [13], a specific recession function was introduced which will play an important role in our study. We set $\Psi(x):=-j^{\circ}(x ;-x)$ and denote by $J_{\infty}^{\circ}$ the recession function associated with $\Psi$, that is,

$$
\begin{aligned}
J_{\infty}^{\circ}(x) & =\liminf _{\substack{\rightarrow \rightarrow \infty \\
y \stackrel{\mathrm{s}}{\rightarrow}}} \Psi(t y) / t=\inf \left\{\liminf _{n \rightarrow \infty} \Psi\left(t_{n} y_{n}\right) / t_{n} \mid t_{n} \rightarrow \infty, y_{n} \stackrel{\mathrm{s}}{\rightarrow} x\right\} \\
& =\inf \left\{\liminf _{n \rightarrow \infty}-j^{\circ}\left(t_{n} y_{n} ;-y_{n}\right) \mid t_{n} \rightarrow \infty, y_{n} \stackrel{\mathrm{s}}{\rightarrow} x\right\} \\
& =\inf \left\{\liminf _{n \rightarrow \infty}-(-j)^{\circ}\left(t_{n} y_{n} ; y_{n}\right) \mid t_{n} \rightarrow \infty, y_{n} \stackrel{\mathrm{s}}{\rightarrow} x\right\} .
\end{aligned}
$$

Brézis \& Nirenberg [7] introduced a recession function associated with a given nonlinear operator $A: V \rightarrow V^{*}$. They defined

$$
\begin{aligned}
r_{A}(u) & :=\liminf \{\langle A(t v), v\rangle \mid t \rightarrow \infty, v \stackrel{\mathrm{s}}{\rightarrow} u\} \\
& =\inf \left\{\liminf _{n \rightarrow \infty}\left\langle A\left(t_{n} v_{n}\right), v_{n}\right\rangle \mid t_{n} \rightarrow \infty, v_{n} \stackrel{\mathrm{s}}{\rightarrow} u\right\} .
\end{aligned}
$$

If we set $\Psi(u):=\langle A u, u\rangle$, then we can see that $r_{A}(u)=\Psi^{\infty}(u)$.

\section{The main result}

Let us introduce the set $R(A, f, j, \Phi, K)$ of asymptotic directions:

$$
\begin{aligned}
R(A, f, j, \Phi, K) & :=\left\{w \in K_{\infty} \mid \exists u_{n} \in K, t_{n}:=\left\|u_{n}\right\| \rightarrow \infty,\right. \\
w_{n} & \left.:=u_{n} /\left\|u_{n}\right\| \rightarrow w \text { and }\left\langle A u_{n}-f, u_{n}\right\rangle-j^{\circ}\left(u_{n} ;-u_{n}\right)+\Phi\left(u_{n}\right) \leq 0\right\} .
\end{aligned}
$$

The study of the properties of the recession set by means of recession tools as those defined in Section 1 constitutes what is called the recession analysis. 
This approach is now the object of intensive work: see Adly, Goeleven \& Théra [1], Attouch, Chbani \& Moudafi [4], Goeleven [11] and the references therein, Goeleven \& Théra [13], F. Tomarelli [26].

TheOrem 4. If the set $R(A, f, j, \Phi, K)$ is empty then H.V.I. $(A, f, j, \Phi, K)$ has at least one solution.

Proof. Set $K_{n}:=\{v \in K \mid\|v\| \leq n\}$. Using Lemma 3, we get the existence of $u_{n} \in K_{n}$ such that

$$
\left\langle A u_{n}-f, v-u_{n}\right\rangle+j^{\circ}\left(u_{n} ; v-u_{n}\right)+\Phi(v)-\Phi\left(u_{n}\right) \geq 0, \quad \forall v \in K_{n} .
$$

Claim 1. There exists $n_{0} \in \mathbb{N}$ such that $\left\|u_{n_{0}}\right\|<n_{0}$.

Indeed, suppose the contrary: $\left\|u_{n}\right\|=n$ for each solution $u_{n}$ of H.V.I. $\left(A, f, j, \Phi, K_{n}\right)$. On relabelling if necessary, we can assume that $w_{n}:=$ $u_{n} /\left\|u_{n}\right\| \rightarrow w$ and

$$
\left\langle A u_{n}-f, v-u_{n}\right\rangle+j^{\circ}\left(u_{n} ; v-u_{n}\right)+\Phi(v)-\Phi\left(u_{n}\right) \geq 0, \quad \forall v \in K_{n} .
$$

By taking $v=0$ in (2.1), we have

$$
\left\langle A u_{n}-f, u_{n}\right\rangle-j^{\circ}\left(u_{n} ;-u_{n}\right)+\Phi\left(u_{n}\right) \leq 0 .
$$

Therefore $w \in R(A, f, j, \Phi, K)$, which contradicts the assumptions of Theorem 4 .

Claim 2. $u_{n_{0}}$ solves H. V.I. $(A, f, j, \Phi, K)$.

Since $\left\|u_{n_{0}}\right\|<n_{0}$, we have, for each $y \in K$, the existence of an $\varepsilon>0$ such that $u_{n_{0}}+\varepsilon\left(y-u_{n_{0}}\right) \in K_{n_{0}}$. It suffices to take

$$
\begin{cases}\varepsilon<\left(n_{0}-\left\|u_{n_{0}}\right\|\right) /\left\|y-u_{n_{0}}\right\| & \text { if } y \neq u_{n_{0}}, \\ \varepsilon=1 & \text { if } y=u_{n_{0}} .\end{cases}
$$

We have

$$
\left\langle A u_{n_{0}}-f, v-u_{n_{0}}\right\rangle+j^{\circ}\left(u_{n_{0}} ; v-u_{n_{0}}\right)+\Phi(v)-\Phi\left(u_{n_{0}}\right) \geq 0, \quad \forall v \in K_{n_{0}} .
$$

If we put $v=u_{n_{0}}+\varepsilon\left(y-u_{n_{0}}\right)$ in $(2.2)$, we obtain

$$
\left\langle A u_{n_{0}}-f, \varepsilon\left(y-u_{n_{0}}\right)\right\rangle+j^{\circ}\left(u_{n_{0}} ; \varepsilon\left(y-u_{n_{0}}\right)\right)+\Phi\left(u_{n_{0}}+\varepsilon\left(y-u_{n_{0}}\right)\right)-\Phi\left(u_{n_{0}}\right) \geq 0 .
$$

Using the convexity of $\Phi$, we derive

$$
\varepsilon\left\langle A u_{n_{0}}-f, y-u_{n_{0}}\right\rangle+\varepsilon j^{\circ}\left(u_{n_{0}} ; y-u_{n_{0}}\right)+\varepsilon\left(\Phi(y)-\Phi\left(u_{n_{0}}\right)\right) \geq 0 .
$$

Dividing (2.3) by $\varepsilon>0$, we finally obtain

$$
\left\langle A u_{n_{0}}-f, y-u_{n_{0}}\right\rangle+j^{\circ}\left(u_{n_{0}} ; y-u_{n_{0}}\right)+\Phi(y)-\Phi\left(u_{n_{0}}\right) \geq 0, \quad \forall y \in K .
$$

This completes the proof. 
We say that $R(A, f, j, \Phi, K)$ is asymptotically compact if the sequence $\left\{w_{n}\right\}_{n \in \mathbb{N}}$ which appears in the definition of this set converges strongly to $w$, that is, if $w_{n}:=u_{n} /\left\|u_{n}\right\|,\left\|u_{n}\right\| \rightarrow \infty, u_{n} \in K$ and

$$
\left\langle A u_{n}-f, u_{n}\right\rangle-j^{\circ}\left(u_{n} ;-u_{n}\right)+\Phi\left(u_{n}\right) \leq 0
$$

implies that $w_{n} \stackrel{\mathrm{s}}{\rightarrow} w$.

Corollary 5. Suppose that the assumptions $(\mathcal{H})$ are satisfied. Assume that:

(i) $R(A, f, j, \Phi, K)$ is asymptotically compact;

(ii) there is a subset $W$ of $V \backslash\{0\}$ such that $R(A, f, j, \Phi, K) \subseteq W$ and

$$
r_{A}(w)+J_{\infty}^{\circ}(w)+\Phi_{\infty}(w)>\langle f, w\rangle, \quad \forall w \in W .
$$

Then the problem H.V.I. $(A, f, j, \Phi, K)$ has at least one solution.

Proof. Suppose by contradiction that $R(A, f, j, \Phi, K)$ is nonempty. Then we can find a sequence $\left\{u_{n}\right\}_{n \in \mathbb{N}}$ such that $t_{n}:=\left\|u_{n}\right\| \rightarrow \infty, w_{n}:=u_{n} / t_{n} \rightarrow w$ and

$$
\left\langle A u_{n}, u_{n}\right\rangle-j^{\circ}\left(u_{n} ;-u_{n}\right)+\Phi\left(u_{n}\right) \leq\left\langle f, u_{n}\right\rangle .
$$

Dividing (2.4) by $t_{n}>0$, we obtain

$$
\left\langle A\left(t_{n} w_{n}\right), w_{n}\right\rangle-j^{\circ}\left(t_{n} w_{n} ;-w_{n}\right)+\frac{\Phi\left(t_{n} w_{n}\right)}{t_{n}} \leq\left\langle f, w_{n}\right\rangle .
$$

Passing to the liminf in (2.5), we derive

$$
r_{A}(w)+J_{\infty}^{\circ}(w)+\Phi_{\infty}(w) \leq\langle f, w\rangle .
$$

Hence, (2.6) contradicts (ii) and the proof follows.

\section{Discussion on a robot hand grasping problem}

Our main motivation in this section is given by a robot hand grasping problem which can be formulated as a variational hemivariational inequality involving monotone and singular matrices. Inequality methods in robotics were introduced by A. M. Al-Fahed, G. E. Stavroulakis \& P. D. Panagiotopoulos [2], P. D. Panagiotopoulos \& A. M. Al-Fahed [22] for the study of unilateral contact effects, both frictionless and frictional ones, between the fingers of a multifingered robotic hand and the manipulated object. In these papers the problem has been reduced to appropriately defined, generally nonsymmetric linear complementarity problems. However, the linear complementarity model is not accurate enough to take into account adhesive effects. More recently, a generalization of the above mentioned model which includes nonmonotone adhesive effects and nonclassical friction effects by including nonconvex yield surfaces in the linear 
complementarity model has been studied by D. Goeleven, G. E. Stavroulakis \& P. D. Panagiotopoulos [12] and G. E. Stavroulakis, D. Goeleven \& P. D. Panagiotopoulos [25]. By applying the theory presented in Section 2 we are able to consider another class of nonmonotone adhesive grippers. We note that the class we will investigate next cannot be considered by means of the theory given in [12]. Conversely, the class of adhesive effects described in [12], [25] is modelled by a specific and complex class of hemivariational inequalities which is not included in the formulation given by H.V.I. $(A, f, j, \Phi, K)$. In this sense, we enlarge the theory applicable to robot hand grasping problems.

Let us consider a rigid object which is grasped by a robotic hand with $n$ elastic fingers. Besides the unilateral contact effects, frictional effects are also assumed on the fingertip-object areas. Only hard fingers which prevent displacements of the object in the normal and tangential directions with respect to the boundary are considered here.

The external forces or torques applied on the reference point of the rigid object are denoted by the vector

$$
p=\left(p_{1}, p_{2}, p_{3}, m_{1}, m_{2}, m_{3}\right)^{t}
$$

(for the general three-dimensional case; in two-dimensional applications $p_{1}$ and $m_{2}, m_{3}$ disappear). With respect to a rectangular cartesian coordinate system $O x_{1} x_{2} x_{3}$, we gather the rigid body displacements and rigid rotations into the vector

$$
u^{0}:=\left(u_{1}^{0}, u_{2}^{0}, u_{3}^{0}, \phi_{1}^{0}, \phi_{2}^{0}, \phi_{3}^{0}\right)^{t},
$$

where the elements $u_{i}^{0}$ denote the rigid body displacements while $\phi_{i}^{0}$ denote the rigid body rotations. Regarding normal forces, they are gathered into the vector

$$
r_{N}:=\left(r_{N_{1}}, \ldots, r_{N_{n}}\right)^{t},
$$

while friction forces and torques are gathered into the vector $r_{T}$ where

$$
r_{T}=\left(r_{T 11}, r_{T 12}, r_{T 21}, r_{T 22}, \ldots, r_{T n 1}, r_{T n 2}\right)^{t} .
$$

As described in [2], [3], the relations that govern the object-gripper system are given by the global equilibrium equations:

$$
G r=G_{N} r_{N}+G_{T} r_{T},
$$

where the equilibrium matrix $G$ is decomposed in a $6 \times n$ submatrix $G_{N}$ for the normal contact reactions and a $6 \times 2 n$ submatrix $G_{T}$ for the frictional forces. Compatibility conditions are given by the formula

$$
u_{N}+d_{N}=u_{N}^{0}
$$


where $u_{N}$ is the deformation of the fingertip, $u_{N}^{0}$ is the normal component of the displacement of the object boundary at points adjacent to the fingertips and $d_{N}$ is the possibly nonzero initial gap between the fingertips and the object.

By means of the principle of complementary virtual work, the relationship is derived as follows: for every statically admissible pair $(p, r)$ we have

$$
p^{t} u^{0}+r^{t} u_{N}^{0}=0
$$

where $u^{0}$ and $u_{N}^{0}$ are supposed to be virtual displacements.

In the direction normal to the surface, the unilateral contact effects between fingertips and object couple $u_{N}$ and $r_{N}$ are described as follows: if contact occurs then a nonnegative reaction arises, that is,

$$
\text { if } u_{N}+d_{N}=u_{N}^{0} \text { then } \quad r_{N} \geq 0,
$$

otherwise if contact is released then a zero reaction must be considered, i.e.

$$
\text { if } u_{N}+d_{N}>u_{N}^{0} \text { then } \quad r_{N}=0 \text {. }
$$

We also assume the existence of a Coulomb law of dry friction connecting the tangential (frictional) forces, exerted by the fingertips on the object with normal (contact) forces. Moreover, to achieve a linear complementarity formulation of the above-described frictional contact gripper, we introduce a piecewise linearization of the friction law by a polyhedral approximation of the friction cone from the interior. We refer to [2] and [3] for more details concerning these considerations.

A linear elastic finger behaviour is assumed next:

$$
u=F r,
$$

where

$$
u=\left(u_{N}, u_{T}\right)^{t}, \quad r=\left(r_{N}, r_{T}\right)^{t}, \quad F:=\left(\begin{array}{cc}
F_{N N} & F_{N T} \\
F_{T N} & F_{T T}
\end{array}\right) .
$$

Here $F$ is the symmetric flexibility matrix which is composed by an $n \times n$ nonsingular normal flexibility matrix $F_{N N}$, the $2 n \times 2 n$ nonsingular tangential flexibility matrix $F_{T T}$ and appropriate couple flexibility matrices $F_{N T}=F_{T N}^{t}$.

Finally, by making use of all these mechanical relations and the unilateral boundary conditions, we obtain the following linear complementarity problem (the complete calculations related to our problem are very long and out of the scope of this paper. A detailed description of this model can be found in A. M. Al-Fahed, G. E. Stavroulakis \& P. D. Panagiotopoulos [3], G. E. Stavroulakis, D. Goeleven \& P. D. Panagiotopoulos [25]):

$$
w-M z=b, \quad w \geq 0, \quad z \geq 0, \quad w^{t} z=0,
$$


with

$$
\begin{aligned}
w & =\left(y_{N}, \gamma\right)^{t}, \quad z=\left(r_{N}, \lambda\right)^{t}, \\
b & =\left(d_{N}-F_{N T}\left(B d_{T}-A^{t} p\right)-G_{N}^{t}\left(A d_{T}-U p\right),-T_{T}^{t}\left(B d_{T}-A^{t} p\right)\right)^{t},
\end{aligned}
$$

and

$$
M:=\left(\begin{array}{cc}
F_{N N}-F_{N T}\left(B F_{T N}+A^{t} G_{N}\right)-G_{N}^{t}\left(A F_{T N}+U G_{N}\right) & T_{N}^{t}-T_{T}^{t}\left(B F_{T N}+A^{t} G_{N}\right) \\
\left(F_{N T} B+G_{N}^{t} A\right) T_{T} & T_{T}^{t} B T_{T}
\end{array}\right)
$$

with

$$
\begin{aligned}
B & :=\left(F_{T T}\right)^{-1}-\left(F_{T T}\right)^{-1} G_{T}^{t}\left(G_{T}\left(F_{T T}\right)^{-1} G_{T}^{t}\right)^{-1} G_{T}\left(F_{T T}\right)^{-1}, \\
A^{t} & :=\left(F_{T T}\right)^{-1} G_{T}^{t}\left(G_{T}\left(F_{T T}\right)^{-1} G_{T}^{t}\right)^{-1}, \\
U & :=-\left(G_{T}\left(F_{T T}\right)^{-1} G_{T}^{t}\right)^{-1} .
\end{aligned}
$$

Here $y_{N}=u_{N}-u_{N}^{0}+d_{N}, \gamma:=\mu\left|r_{N}\right|-\left|r_{T}\right|, \mu$ is the coefficient of friction, $\lambda$ is the nonnegative slipping value associated with $\gamma, d_{T}$ is the initial tangential distance between the fingertip and the potential point of contact, the matrices $T_{N}$ and $T_{T}$ are the matrices of the linearized friction law (here for a vector $x=\left\{x_{i}\right\}$, we denote by $|x|$ the vector $\left.\left\{\left|x_{i}\right|\right\}\right)$. The linear complementarity problem is defined on $K:=\mathbb{R}_{+}^{n+m}$. Here $m$ is equal to $n \times l$ for the hard-finger model where $l$ is the number of the facets of the linearized friction cone [2]. It is known [2] that $M$ is a positive semidefinite matrix.

We now extend the previous model to cover nonclassical fingertip-object interaction effects by using the theory derived in Section 2. The possibility of existing adhesive and unilateral effects with nonconvex yielding characteristics on the fingertips will be included in our previous model (unilateral contact part and adhesive part will be denoted by subscripts "u" and "a" respectively). In the spirit of a decomposition in unilateral and adhesive parts, the notation $y_{N_{\mathrm{u}}}$, $r_{N_{\mathrm{u}}}, \gamma_{\mathrm{u}}, \lambda_{\mathrm{u}}$ should be used in our previous relations. Nevertheless, for notational simplicity, we avoided showing all these indices untill now that the classical frictional contact problem has been formulated. We know that the unilateral effects can be described by a linear complementarity problem which is also equivalent to the following variational inequality:

$$
z_{\mathrm{u}} \in K: \quad w_{\mathrm{u}}^{t}\left(v-z_{\mathrm{u}}\right) \geq 0, \quad \forall v \in K .
$$

Let us consider the case where adhesive effects can be represented by nonconvex yield surfaces in the $\left(r_{N}, \lambda\right)$ space. Let

$$
r_{N}=r_{N_{\mathrm{u}}}, \quad y_{N}=y_{N_{\mathrm{u}}}+y_{N_{\mathrm{a}}}, \quad \lambda=\lambda_{\mathrm{u}}, \quad \gamma=\gamma_{\mathrm{u}}+\gamma_{\mathrm{a}} .
$$

Let $y_{N_{\mathrm{a}}}$ and $\gamma_{\mathrm{a}}$ be derived by

$$
w_{\mathrm{a}}=w_{\mathrm{a}, 1}+w_{\mathrm{a}, 2},
$$


where

$$
\begin{aligned}
& w_{\mathrm{a}, 1} \in-\partial \Phi\left(r_{N}, \lambda\right), \\
& w_{\mathrm{a}, 2} \in-\bar{\partial} j\left(r_{N}, \lambda\right) .
\end{aligned}
$$

Here $\Phi: \mathbb{R}^{n+m} \rightarrow \mathbb{R}$ is a proper convex and lower semicontinuous functional and $\partial \Phi$ denotes its convex subdifferential and $j: \mathbb{R}^{n+m} \rightarrow \mathbb{R}$ is a locally Lipschitz functional and $\bar{\partial} j$ denotes its Clarke's subdifferential. Law (3.2) contains as a special case the case of adhesive rotational contact, as well as the case of zigzag rotational and tangential law. See P. D. Panagiotopoulos [21] and J. J. Moreau \& P. D. Panagiotopoulos [18] for more details. Law (3.2) is a well-known convex superpotential [17] which can be used to describe monotone tangential law.

We have

$$
\Phi(v)-\Phi(z)+w_{\mathrm{a}, 1}^{t}(v-z) \geq 0, \quad \forall v \in \mathbb{R}^{n+m},
$$

and

$$
j^{\circ}(z ; v-z)+w_{\mathrm{a}, 2}^{t}(v-z) \geq 0, \quad \forall v \in \mathbb{R}^{n+m} .
$$

We have

$$
w^{t}(v-u)=w_{\mathrm{a}}^{t}(v-u)+w_{\mathrm{u}}^{t}(v-u)
$$

and thus our problem can be described by the following variational hemivariational inequality:

(3.4) $z \in K: \quad(M z-b)^{t}(v-u)+\Phi(v)-\Phi(u)+j^{\circ}(u ; v-u) \geq 0, \quad \forall v \in K$.

It is clear that $R(M, b, j, \Phi, K)$ is asymptotically compact since our problem is stated in a finite-dimensional space. Moreover, we can prove (see [13] for more details) that

$$
R(M, b, j, \Phi, K) \subseteq \operatorname{Ker}\left(M+M^{t}\right) \cap K \backslash\{0\} .
$$

Therefore, a sufficient condition for the solvability of problem (3.4) is

$$
\langle b, e\rangle<\Phi_{\infty}(e)+J_{\infty}^{\circ}(e), \quad \forall e \in \operatorname{Ker}\left(M+M^{t}\right) \cap K \backslash\{0\} .
$$

REMARK 6 .

(i) For a thorough discussion concerning the role of such a condition in mechanics we refer to [1], [4], [5], [11], [25].

(ii) Several results concerning the estimation of the recession functional $J_{\infty}^{\circ}$ are stated in [13].

\section{REFERENCES}

[1] S. Adly, D. Goeleven And M. ThéRA, Recession mappings and noncoercive variational inequalities, Nonlinear Anal. (to appear). 
[2] A. M. Al-Fahed, G. E. Stavroulakis and P. D. Panagiotopoulos, Hard and soft fingered robot grippers. The linear complementarity approach, Z. Angew. Math. Mech. 71 (1991), 257-265.

[3] - A linear complementarity approach to the frictionless gripper, Internat. J. Robot. Res. 11 (1992), 112-122.

[4] H. Attouch, Z. Chbani And A. Moudafi, Recession operators and solvability of variational problems, preprint, Laboratoire d'Analyse Convexe, Université de Montpellier II, 1993.

[5] C. Baiocchi, G. Buttazzo, F. Gastaldi and F. Tomarelli, General existence theorems for unilateral problems in continuum mechanics, Arch. Rational Mech. Anal. 100 (1988), 149-180.

[6] H. Ben-El-Mechaiekh, P. Deguire et A. Granas, Une alternative non linéaire en analyse convexe et applications, C. R. Acad. Sci. Paris 295 (1982), 382-384.

[7] H. Brézis And L. Nirenberg, Characterizations of ranges of some nonlinear operators and applications to boundary value problems, Ann. Scuola Norm. Sup. Pisa Cl. (4) 5 (1978), 225-325.

[8] F. H. Clarke, Optimization and Nonsmooth Analysis, Wiley, 1983.

[9] K. FAn, A minimax inequality and applications, Inequalities III (O. Shisha, ed.), Academic Press, New York and London, 1972, pp. 103-113.

[10] D. Goeleven, On a class of hemivariational inequalities involving hemicontinuous monotone operators. Applications in unilateral mechanics, FUNDP Research report, 1994.

[11] Noncoercive variational problems. The recession analysis, FUNDP Research report, 1995.

[12] D. Goeleven, G. E. Stavroulakis and P. D. Panagiotopoulos, Solvability theory for a class of hemivariational inequalities involving copositive plus matrices. Application in robotics, preprint, 1995.

[13] D. Goeleven And M. ThÉRA, Semicoercive variational hemivariational inequalities, J. Global Optim. (special issue: Nonconvex energy functions. Applications in engineering) 6 (1995), 367-381.

[14] A. Granas, Sur quelques méthodes topologiques en analyse convexe, Variational Methods in Nonlinear Problems, part 3, Les Presses de l'Université de Montréal, 1990.

[15] V. Kumar and K. J. Waldron, Suboptimal algorithms for force distribution in multifingered grippers, IEEE Trans. Robot. Automat. 5 (1989), 491-498.

[16] E. N. Mitsopoulos And I. N. Doudoumis, A contribution to the analysis of unilateral contact problems with friction, Solid Mech. Arch. 12 (1987), 165-186.

[17] J. J. Moreau, La notion de sur-potentiel et les liaisons unilatérales en élastostatiques, C. R. Acad. Sci. Paris Sér. A 267 (1968), 954-957.

[18] J. J. Moreau and P. D. Panagiotopoulos (eds.), Nonsmooth Mechanics and Applications, CISM Lecture Notes, vol. 302, Springer-Verlag, Wien, 1988.

[19] Z. Naniewicz and P. D. Panagiotopoulos, The Mathematical Theory of Hemivariational Inequalities and Applications, Marcel Dekker, New York, 1994.

[20] P. D. Panagiotopoulos, Hemivariational Inequalities, Applications in Mechanics and Engineering, Springer-Verlag, Berlin, 1993.

[21] Inequality Problems in Mechanics and Applications, Convex and Nonconvex Energy Functions, Birkhäuser, Basel, 1985.

[22] P. D. Panagiotopoulos And A. M. Al-Fahed, Robot hand grasping and related problems: Optimal control and identification, Internat. J. Robot. Res. 13 (1994), 127136. 
[23] R. T. Rockafellar, Convex Analysis, Princeton University Press, Princeton, N.J., 1970.

[24] S. Simons, Two-function minimax theorems and variational inequalities for functions on compact and noncompact sets, with some comments on fixed-point theorems, Proc. Sympos. Pure Math., vol. 45, Amer. Math. Soc., 1986, pp. 377-392.

[25] G. E. Stavroulakis, D. Goeleven and P. D. Panagiotopoulos, New models for a class of adhesive grippers. The hemivariational inequality approach, preprint, 1995.

[26] F. Tomarelli, Noncoercive variational inequalities for pseudomonotone operators, preprint, 1993.

[27] C. L. Yen, A minimax inequality and its applications to variational inequalities, Pacific J. Math. 97 (1977), 477-481.

SAmir Adly and Michel ThÉRA

LACO, URA-CNRS 1586

Université de Limoges

87060 Limoges Cedex, FRANCE

E-mail address: adly@unilim.fr thera@cix.cict.fr

\section{Daniel Goeleven}

FNRS

Facultés Notre Dame de la Paix

B-5000 Namur, BELGIQUE

E-mail address: dgoeleven@cc.fundp.ac.be 